\title{
FISIOLOGÍA \\ del Ciclo Estral Bovino
}

Colazo, M.G. ${ }^{1}$ y Mapletoft, R.J. ${ }^{1}$

\section{Introducción}

U

na mejor comprensión de la fisiología del ciclo estral y la función ovárica ha resultado en una mayor capacidad para manipular su control. Descubrimientos recientes de las funciones del cuerpo lúteo y las ondas foliculares del ciclo estral bovino se han traducido en un renovado entusiasmo por las oportunidades de poder lograr un mejor control de la inducción de la ovulación y una más precisa sincronización del ciclo estral. El alto nivel de interés se refleja en el gran aumento de revisiones publicadas sobre la manipulación del ciclo estral durante los últimos ańos (Odde, 1990; Larson y Ball, 1992; Wiltbank, 1997; Roche et al., 1997; Mapletoft et al., 2002; Macmillan et al., 2003; Kastelic et al., 2008; Macmillan, 2010; Colazo y Mapletoft 2014). La intención de este manuscrito es proporcionar una visión general de los eventos ováricos normales en el ganado bovino adulto y prepúber, con la idea de impulsar una discusión para aumentar aun más nuestra capacidad de manipulación del ciclo estral bovino.

\section{Regulación endócrina del ciclo estral}

Los bovinos son animales poliéstricos con ciclos estrales cada 21 días (rango 17-24 días) en promedio. El ciclo estral está regulado por las hormonas del hipotálamo (hormona liberadora de gonadotrofina, GnRH), la pituitaria anterior (hormona folículo estimulante, FSH y hormona luteinizante, LH), los ovarios (progesterona, P4; estradiol, E2 e inhibinas) y el útero (prostaglandina F2 $\alpha$, PGF). Estas hormonas actúan a través de un sistema de retroalimentación positiva y negativa para gobernar el ciclo estral del bovino (Stevenson, 2007). La GnRH es un decapéptido producido en las neuronas del área ventromedial y del área preóptica del hipotálamo. La GnRH es secretada de dos formas: una secreción pulsátil

1 Livestock Research Branch, Alberta Agriculture and Rural Development, Edmonton, Alberta, Canada. WCVM, University of Saskatchewan, Saskatoon, Saskatchewan, Canada. marcos.colazo@gov.ab.ca. 
o tónica desde el centro tónico del hipotálamo y la secreción preovulatoria de GnRH que anteriormente se creía que era directamente estimulada por el E2. Sin embargo ahora se conoce que las neuronas secretoras de GnRH no tienen receptores para E2 por lo que una acción directa sobre la secreción de $\mathrm{GnRH}$ es poco probable. Existe un grupo de neuronas hipotalámicas que expresan el gen Kiss-1 que codifica el péptido Kisspeptina. Se ha demostrado que este péptido es un potente estimulador de la secreción de $\mathrm{GnRH}$ y que las neuronas secretoras de $\mathrm{GnRH}$ tienen receptores para este péptido (Gottsch et al., 2004). Por lo tanto se cree que la Kisspeptina podría proveer información a las neuronas secretoras de GnRH con respecto a las concentraciones sanguíneas de las hormonas esteroides.

La GnRH llega a la pituitaria anterior a través del sistema portahipotálamo-hipofisiario (Moenter et al., 1992) y controla la liberación de LH y FSH uniéndose a su proteína G acoplada al receptor en la superficie celular de las células gonadotrofos (Kakar et al., 1993). La FSH sólo se almacena en gránulos secretores en el citoplasma durante períodos cortos de tiempo, mientras que la LH se almacena durante períodos más largos durante el ciclo estral (Farnwort, 1995).

Durante la fase folicular del ciclo estral las concentraciones de P4 circulante son bajas debido a la regresión del cuerpo lúteo (CL). El aumento de las concentraciones de E2, proveniente del folículo dominante preovulatorio induce un pico de GnRH (a través de la Kisspeptina) y a su vez permite la visualización del comportamiento estral durante el cual las hembras son sexualmente receptivas y permiten ser montadas (Stevenson, 2007). Este pico preovulatorio de GnRH induce un pico de LH y FSH (Nett et al., 1984) y la ovulación ocurre en promedio a las 27 horas después del pico de LH o inicio del estro. La ovulación es seguida por la fase lúteal del ciclo estral. Los primeros 3-4 días son conocidos como el metaestro que es cuando toma parte la formación del CL (llamado en este momento cuerpo hemorrágico). En los días siguientes (diestro) la concentración de P4 en sangre comienza a aumentar debido a la formación del CL en el que las células lutenizadas de la granulosa y la teca producen grandes cantidades de P4 en preparación para el establecimiento y mantenimiento de la preñez o la reanudación del ciclo estral (Niswender et al., 2000). Durante el diestro hay crecimiento folicular en el ovario pero como veremos más adelante estos folículos no ovulan ya que la $\mathrm{P} 4$ a través de una retroalimentación negativa sobre la GnRH, sólo permite la secreción de pulsos de LH de mayor amplitud pero menor frecuencia (1 pulso cada 3-4 horas) que son inadecuados para la ovulación del folículo dominante (Rahe et al., 1980). 
Después de un período de 12-14 días de exposición a altos niveles séricos de P4, el CL regresa en respuesta a la secreción de PGF del útero que llega al ovario a través de un mecanismo de contra-corriente (Ginther, 1974), y da lugar al inicio del proestro. El proestro dura 2-3 días y es caracterizado por un incremento en la frecuencia de los pulsos de LH (1 pulso cada hora) que conducen a la maduración final del folículo ovulatorio y al incremento del E2 que desencadena el comportamiento sexual (estro) en el bovino.

\section{La dinámica folicular y luteal durante el ciclo estral I}

\section{Las ondas foliculares}

El primero en postular la teoría de las ondas foliculares fue Rajakoski en su publicación del año 1960 (Rajakoski, 1960). Sin embargo no fue hasta en la década del 80 , cuando la ecografía se empezó a utilizar como un método de estudio de la función ovárica en el ganado bovino (Pierson y Ginther, 1984), que se demostro que más del $95 \%$ de los ciclos estrales se componen de 2 o 3 ondas foliculares (Figura 1; Ginther et al., 1989a; 1989c; Savio et al., 1988; Sirois y Fortune, 1988; Knopf et al., 1989). Ciclos estrales de una onda folicular han sido reportados en novillas en el momento de la pubertad (Evans et al., 1994a) y en vacas maduras durante el primer intervalo interovulatorio después del parto (Murphy et al., 1990; Savio et al., 1990a; 1990b). Ciclos estrales de cuatro ondas se observan ocasionalmente en Bos indicus (Rhodes et al., 1995; Zeitoun et al., 1996), pero los ciclos estrales compuestos por 4 o más ondas foliculares son acompańados por un intervalo interovulatorio prolongado como consecuencia del retraso en la luteólisis o una falla de ovulación (Ko et al., 1991; Adams et al., 1992a; Roche y Boland, 1991). La proporción de animales con 2 vs. 3 ondas foliculares varía entre informes, algunos autores reportaron que la mayoría de los ciclos fueron de 2 ondas (>80\%, Ginther et al., 1989c; Rajamahendran y Taylor, 1990), otros informaron una mayoría de 3 ondas ( $>80 \%$, Sirois y Fortune, 1988), mientras que otros han observado una distribución más uniforme (Evans et al., 1994; Savio et al., 1990a; 1993a). Rhodes et al. (1995) evaluó 117 intervalos inter-ovulatorios de 17 vaquillas Brahman y reporto que el $26 \%, 68 \%$ y $7 \%$ de los ciclos estrales estaban compuestos de 2, 3 y 4 ondas foliculares, respectivamente.

Aunque el tema no se ha estudiado de forma sistemática, no parece haber ninguna clara preferencia de raza o edad específica para un patrón de onda 
folicular sobre el otro. En términos generales en los animales Bos indicus mayor cantidad de folículos son reclutados en cada onda folicular y tienen más ondas foliculares por ciclo estral. El diámetro del folículo ovulatorio es mas pequeńo y consecuentemente el tamańo del CL y la concentración de P4 circulante serán menores (Sartori y Barros, 2011).

Al parecer tampoco hay ninguna diferencia aparente en la fertilidad de animales con diferentes patrones de desarrollo folicular. Dos estudios publicaron que la tasa de concepción al primer servicio se redujo en vacas en las que el folículo ovulatorio provenía de la segunda onda folicular en comparación con vacas que ovularon el folículo dominante de la tercera onda (Ahmad et al., 1997; Townson et al., 2002). Sin embargo en otro estudio, la tasa de preñez no difirió entre vacas lecheras en lactancia con diferentes patrones de ondas de desarrollo folicular, aunque la fertilidad se correlacionó negativamente con el intervalo entre la emergencia de la onda folicular y el celo (Bleach et al., 2004).

En un estudio sobre los efectos de la nutrición en la dinámica folicular (Murphy et al., 1991), vacas alimentadas con una ración de baja energía tuvieron una mayor proporción de ciclos de 3 ondas foliculares que aquellas alimentadas con raciones de alta energía. Datos preliminares obtenidos de 9 novillas durante sus primeros 2 años de vida sugieren que el patrón se puede repetir dentro de los individuos (Dr. Gregg Adams comunicación personal). Es decir, un patrón de 2 ondas es más probable que sea seguido por un patrón de 2 ondas, y uno de 3 seguido de otro de 3 ondas. La razón evolutiva de un ciclo de 2 o 3 ondas, o el hecho en sí de un crecimiento folicular en ondas no está bien claro, pero las diferencias entre los patrones de crecimiento de las ondas foliculares tienen consecuencias claras con respecto a los protocolos para la sincronización de la ovulación y superestimulación ovárica.

\section{Patrón de crecimiento del desarrollo de folículos antrales}

El patrón de crecimiento del desarrollo de folículos antrales en los ovarios se ha dividido arbitrariamente en dos fases distintas (Mihm y Bleach, 2003). Una fase de crecimiento "lenta" que es desde la adquisición del antro hasta un tamaño detectable mediante exámenes ecográficos $(3 \mathrm{a} 4 \mathrm{~mm}$ ) y la fase de crecimiento "rápida" desde el momento de la emergencia de la onda folicular a la ovulación o la atresia del folículo dominante. El crecimiento de los folículos antrales ováricos desde la adquisición de un antro (0.3 $\mathrm{mm}$ ) a un diámetro de entre 3 a $4 \mathrm{~mm}$ (fase de crecimiento "lenta") lleva 
más de 30 días (Lussier et al., 1987). En el ganado bovino, todavía no está bien claro si esta etapa del desarrollo folicular es posible sin la hormona FSH. Sin embargo, existen evidencias de que los receptores de FSH están presentes (Xu et al., 1995; Bao y Garverick, 1998) y funcionalmente activos (McNatty et al., 1999) durante el desarrollo preantral y antral temprano en el bovino, lo que sugiere que la FSH podría tener un papel importante en las primeras etapas del desarrollo folicular. Además, los folículos de lauchas sin la capacidad de responder a la FSH no muestran progresión desde etapas preantrales a estadios antrales tempranos del desarrollo folicular (Abel et al., 2000). Por el contrario, es bien sabido que los folículos en la fase de "rápido" crecimiento son absolutamente dependiente de las concentraciones adecuadas de FSH y LH (Ginther et al., 1996a; 1996b).

El patrón de la onda de desarrollo folicular se refiere simplemente al periódico, y sincrónico crecimiento de un grupo de folículos antrales. El patrón de la onda de desarrollo folicular se ha demostrado en todas las especies en las que se ha examinado, incluyendo ovejas (Ravindra et al., 1994), cabras (Ginther y Kot, 1994), yeguas (Ginther, 1993), camélidos (Adams et al., 1990), y algunos ungulados silvestres (Hoare et al., 1997). En el ganado bovino, la emergencia de la onda se caracteriza por el repentino (dentro de 1 a 2 días) crecimiento de un numero variable (de 8 a 41) de folículos que se detectan inicialmente por ultrasonografía cuando tienen un diámetro de 3 a $4 \mathrm{~mm}$ (Ginther et al., 1989a). En los primeros 2 días, la tasa de crecimiento es similar entre todos los folículos de una onda, luego se seleccionará 1 folículo que continuará el crecimiento (folículo dominante) mientras que el resto de los folículos (folículos subordinados) se atresian. En ciclos estrales de 2 y 3 ondas, la aparición de la primera onda folicular ocurre constantemente en el día de la ovulación (que es comúnmente designado como Día 0). La aparición de la segunda onda se produce en el Día 9 o 10 en los animales con ciclos de 2 ondas, y en el día 8 o 9 en los animales con ciclos de 3 ondas (es decir, 1 o 2 días antes que los de 2 ondas). En los ciclos de 3 ondas, una tercera onda emerge en el Día 15 o 16. Las ondas foliculares sucesivas permanecerán anovulatorias hasta que ocurra la luteólisis (Bergfelt et al., 1991). El folículo dominante presente en el inicio de la luteólisis se convertirá en el folículo ovulatorio, y la aparición de la siguiente onda se retrasa hasta el día de la ovulación (Día 0). El cuerpo lúteo comienza a regresar más temprano en animales con ciclos de 2 ondas (Día 16) que en los animales con ciclos de 3 ondas (Día 19), resultando en un ciclo estral correspondientemente más corto (20 vs. 23 días, respectivamente). Por lo tanto, en realidad el ciclo de libro 
de 21 días es muy raro, es solamente un promedio entre ciclos estrales de 2 y 3 ondas foliculares.

\section{Papel de las gonadotrofinas otros factores en el desarrollo de la onda folicular}

La naturaleza ha desarrollado una estrategia (en el caso del bovino) que le permite a un folículo seguir creciendo y tener el potencial de ovular, mientras que al mismo tiempo minimiza el desgaste de folículos de la reserva al no permitir el reclutamiento folicular entre ondas foliculares. El mecanismo se basa en la capacidad de respuesta diferencial a las gonadotrofinas, FSH y LH (Ginther et al., 1996b). Aumentos periódicos en las concentraciones de FSH circulantes son responsables de inducir las emergencias de las ondas foliculares, por lo tanto las vacas con 2 ondas tienen 2 picos de FSH y las vacas con 3 ondas tienen 3 picos (Figura 1; Adams et al., 1992b). La FSH circulante es posteriormente suprimida por el feedback negativo de los productos de los folículos emergentes (principalmente E2 e inhibina) y el mantenimiento de una concentración de FSH baja previene eficazmente la emergencia de una nueva onda folicular (Adams et al., 1992a). Como dicho anteriormente, la supresión periódica de FSH conserva los recursos del ovario al impedir el reclutamiento continuo de folículos antrales, el 99\% de los cuales se pierden en la atresia. El aumento transitorio de FSH permite suficiente crecimiento folicular para que algunos (no todos) folículos adquieran capacidad de respuesta a la LH. En otras palabras, algunos folículos adquieren la capacidad de sobrevivir sin FSH (Ginther et al., 1996b). En el momento en que los perfiles de crecimiento del folículo dominante y folículos subordinados comienzan a divergir (momento de la selección o divergencia), aproximadamente 2.8 días después de la emergencia de la onda (cuando el futuro folículo dominante tiene $8.5 \mathrm{~mm}$ de diámetro in Bos taurus o aproximadamente $6.2 \mathrm{~mm}$ in Bos indicus), la FSH disminuye rápidamente. El folículo destinado a convertirse en dominante parece tener más receptores de LH en las células de la ganulosa y por eso tiene la ventaja competitiva sobre los folículos destinados a convertirse en subordinados. Sin embargo, la capacidad de respuesta a la LH y la capacidad de convertirse en un folículo dominante no es un proceso de todo o nada, y probablemente representa una diferencia cuantitativa más que una diferencia absoluta entre los folículos de una onda. Los folículos subordinados pueden llegar a ser dominantes si el original folículo dominante se remueve (Ko et al., 1991; Adams et al., 1993b) o si se suministra FSH exógena en el momento de la 
deviación folicular (Adams et al., 1993a). Además, la competencia para la LH entre múltiples folículos dominantes (es decir, en animales tratados con FSH) se puede comprobar por el menor diámetro máximo alcanzado en estos folículos en comparación con folículos dominantes individuales (Adams et al., 1993a).

Cada vez hay más pruebas de que el sistema de factores de crecimiento similares a la insulina (IGF-I) puede jugar un papel crítico en la selección del folículo dominante (Fortune et al., 2001). Estos factores mantienen el crecimiento folicular por estímulo de la proliferación de las células de la granulosa y junto a las gonadotrofinas promueven la diferenciación de las células de la gránulosa y de la teca (Spicer y Echternkamp, 1995). Por lo tanto, aquellos folículos con mayores concentraciones de IGF disponibles en el líquido folicular son más propensos a convertirse en dominantes. Interesantemente, cambios en los componentes intrafoliculares del sistema de IGF-I, en particular las concentraciones de proteínas de bajo peso moleculares que fijan IGF-I (IGFBP; insulin growth factor binding protein) o proteasas (PAPP-A; pregnancy-associated plasma protein-A) que degradan las IGFBP parecen estar estrechamente relacionadas con la selección del futuro folículo dominante (Fortune et al., 2001). En este sentido, Mihm y colaboradores (2000) informaron de que sobre la base de muestras de líquido folicular bovino tomadas en el día 1.5 de la primera onda folicular, el folículo con la concentración más baja de IGFBP-4 siempre se convirtió en el folículo dominante. Además, Armstrong y colaboradores (1998) informaron de que la concentración intrafolicular de IGFBP-2 fue menor en el folículo dominante cuando se comparó con la concentración en el folículo subordinado.

Una continua supresión de LH como consecuencia de un aumento de secreción de la progesterona durante la fase luteal provoca que el folículo dominante cese sus funciones metabólicas y comience a morir. Tras el cese de la secreción de los productos foliculares por parte del folículo dominante, la FSH circulante comienza a aumentar nuevamente. Este aumento no tiene ningún efecto sobre el folículo dominante que está regresando, pero es responsable de provocar la emergencia de una nueva onda folicular.

Al final de la fase luteal, una disminución de la concentración sanguínea de progesterona (debido a la luteólisis provocada por la liberación de PGF) permite que la frecuencia de los pulsos de LH aumente, estimulando así un mayor crecimiento del folículo dominante y aumento de la secreción de E2, que finalmente resultara en un pico de LH seguido de la ovulación. Por lo tanto el ciclo estral se repite. 


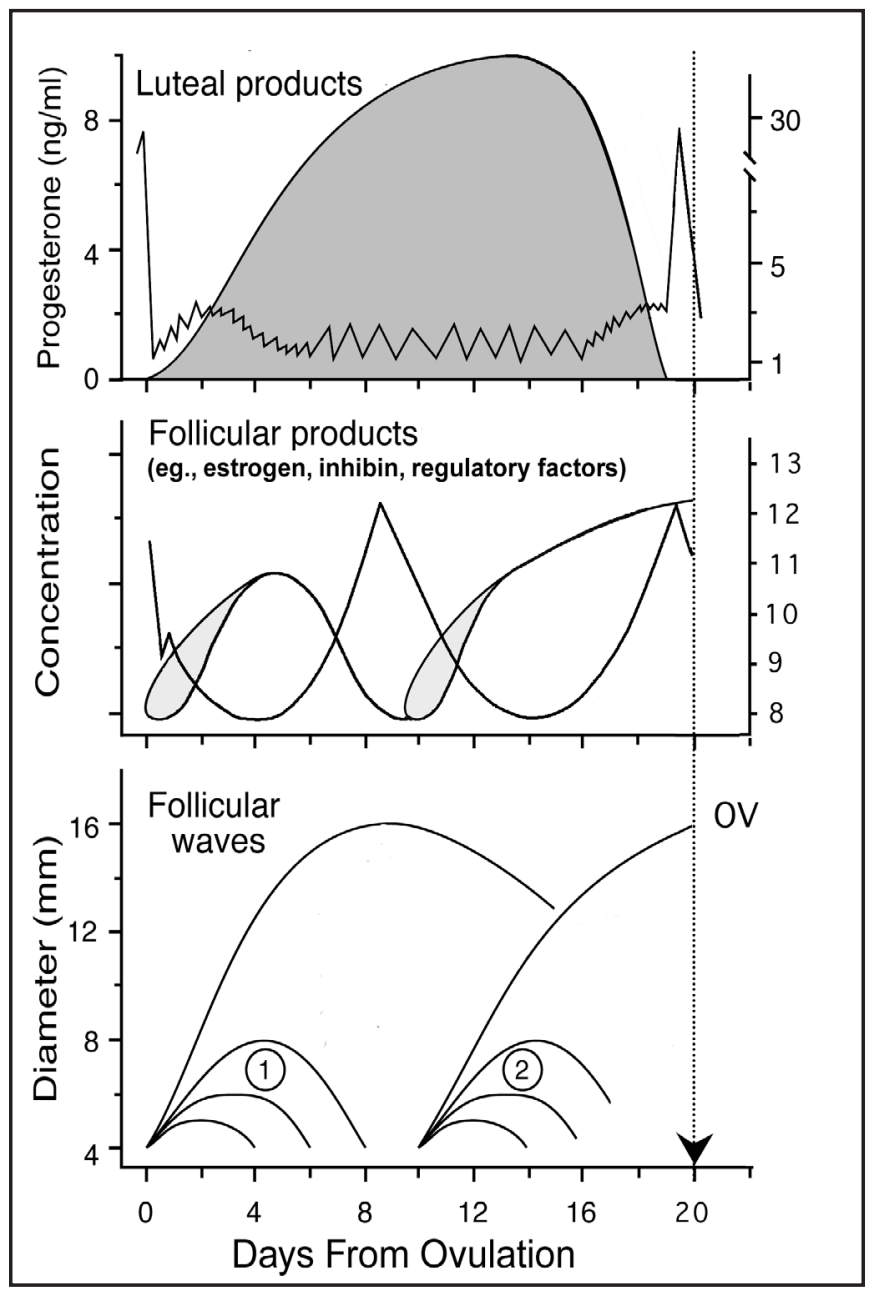



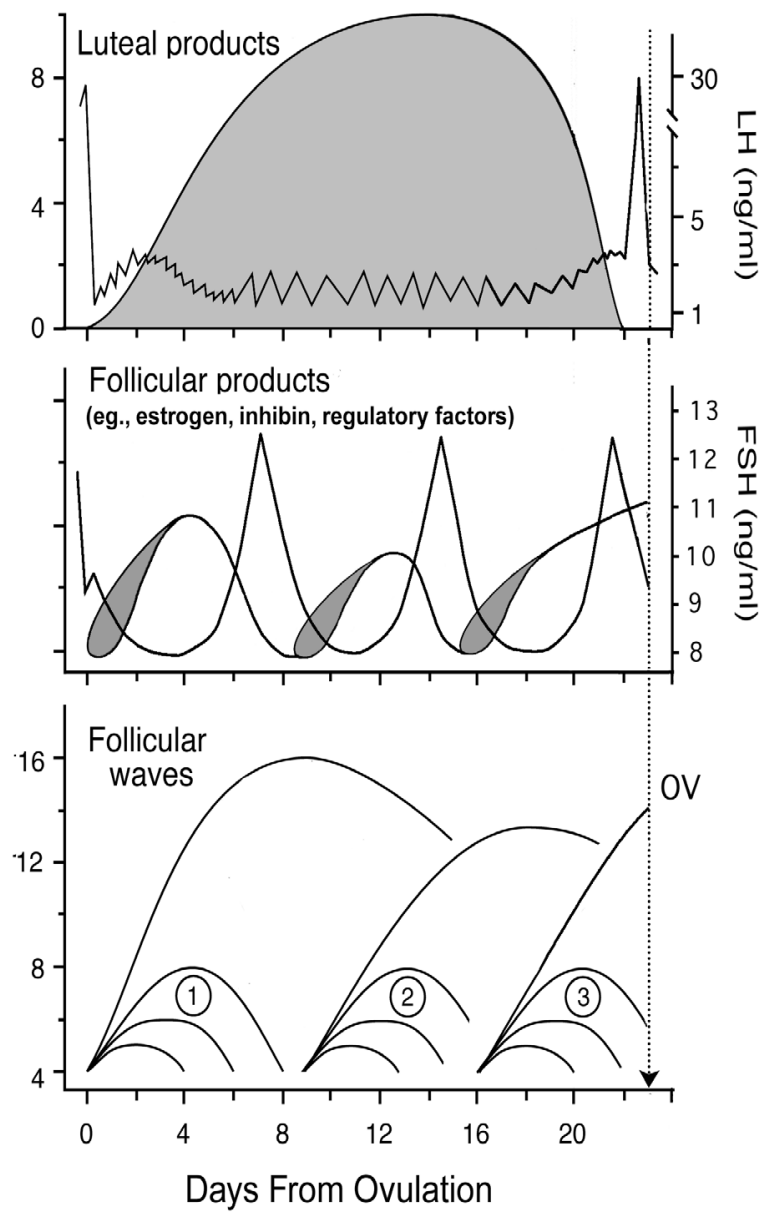

Figura 1. Dinámica ovárica durante los ciclos de 2 y 3 ondas foliculares en el ganado bovino ( $\mathrm{OV}$ = ovulación). Cuando la concentración de progesterona (área en gris) es baja, la frecuencia de pulsos de LH (línea) es alta; cuando los niveles de progesterona están altos, la frecuencia de pulsos de LH es baja y la amplitud de los mismos es grande. Las formas que representan los productos foliculares corresponden al número relativo de folículos que contribuyen a la producción de esos productos en un momento dado. Las líneas representando las ondas foliculares muestran el crecimiento y regresión de los folículos subordinados y el folículo dominante. Los pulsos episódicos de LH son esquemáticos. Figura adaptada de Adams et al., $1992 \mathrm{~b}$. 


\section{El desarrollo folicular durante el puerperio}

- Vacas lecheras

La intensificación de desarrollo de las ondas foliculares ocurre temprano en el período posparto, tanto en el ganado lechero como en el de carne. La aparición de la primera onda folicular posparto varió de 2 a 7 días (promedio 4.0 días) después del parto en vacas Holstein de primer parto (Ginther et al., 1996a), y el folículo dominante de la primera onda posparto ovulo en el 54\% (148/272) de las vacas Holstein primíparas y multíparas en el día 20.4 de promedio (rango, 10 a 36; Colazo datos no publicados). La primera ovulación no fue acompańada por un comportamiento estral en 17 de 18 (94\%) vacas después del parto (Savio et al., 1990a), y la longitud del primer intervalo interovulatorio posparto fue variable dependiendo de cuando el folículo destinado a ovular había surgido. El primer intervalo interovulatorio posparto fue corto (media de 11.2 días) en aproximadamente el 25\% de las vacas lecheras, fue de duración normal (media de 20.6 días) en otro 25\%, y fue considerado largo (media, 30.0 días) en el 50\% de las vacas restantes (Savio et al., 1990a). Los ciclos cortos se asociaron con una detección posterior del primer folículo destinado a ovular (es decir, $\geq 10$ días después del parto), mientras que los ciclos normales y los largos fueron asociados a la detección temprana del primer folículo destinado a ovular (es decir, por lo general $\leq 10$ días después del parto). Estos últimos resultados son muy consistentes con los de otro estudio (Rajamahendran y Tayor, 1990) en la que los intervalos de parto a primera ovulación (media, 21 días; rango, 10-55 días) y primer estro (media, 59 días, rango 17-139 días) no fueron diferentes entre las vacas lecheras primíparas y multíparas. Además, estos autores también reportaron que los períodos anovulatorios posparto cortos (alrededor de 14 días) fueron seguidos por ciclos de longitud normal (18 - 21 días), mientras que períodos más largos (anovulación posparto de 21 a 25 días) fueron seguidos por ciclos cortos (< 14 días). Los ciclos cortos se asociaron con fases luteales más cortas, un CL de menor tamaño, y concentraciones de progesterona circulantes inferiores.

- Vacas de carne

La lactancia y la succión por parte del ternero parecen tener un efecto supresor sobre el desarrollo folicular en el ovario. Los efectos supresores de la succión sobre la ciclicidad estral en el ganado de carne se conoce desde 
hace mucho tiempo, pero la dinámica folicular en vacas de cría se ha caracterizado recientemente (Murphy et al., 1990). En el ganado de carne, al igual que en el ganado lechero, el comienzo de las ondas foliculares se observan dentro de los 10 días después del parto. Sin embargo, la primera ovulación se produce más tarde que en el ganado lechero (promedio 30.6 días) y sólo en raras ocasiones el folículo dominante de la primera es el que ovula (11\%; Murphy et al., 1990). En la mayoría de las vacas (78\%), la ovulación se produjo a partir del folículo dominante de la segunda, tercera o cuarta onda folicular posparto, y como en el ganado lechero, primo ovulaciones que ocurrieron después de los 20 días (16/18 vacas) fueron seguidas por un ciclo corto (14/16 vacas). El destete temporario del ternero (durante horas o días) produce un aumento marcado de las concentraciones circulantes de LH y una aceleración de la aparición del, pero no se ha publicado una comparación directa de la dinámica folicular entre el ganado que es amamantado y el que no lo es. Las diferencias entre las vacas lecheras y vacas de carne en cuanto a la función ovárica posparto parecen diferencias en magnitud en lugar de diferencias en esencia. La reanudación de la ciclicidad ovulatoria un poco antes en vacas lecheras comparado con vacas de carne puede ser reflejo de una mayor presión de selección para esta característica, o simplemente, la falta de amamantamiento.

\section{Priming de progesterona}

La exposición a niveles elevados de progesterona parece ser un requisito previo para la expresión normal del estro y para el desarrollo de una fase lúteal normal. Ciclos estrales cortos pueden ser provocados por la ovulación inducida por GnRH durante el anestro en el ovino y bovino (Troxel y Kesler, 1984), pero ciclos normales pueden lograrse dando progesterona exógena antes del tratamiento con GnRH (Smith et al., 1987). Por lo tanto, la exposición a progesterona seguida por una disminución en las concentraciones circulantes de progesterona (llamado priming de progesterona) parece ser un requisito necesario para la diferenciación normal de las células de la granulosa y el desarrollo del cuerpo luteo después de la ovulación. Probablemente, el mecanismo asociado implica los efectos de un aumento de los impulsos de la frecuencia de LH sobre la producción de estrógeno folicular, el desarrollo de los receptores de LH y la luteinización (Inskeep et al., 1988). La asociación entre los ciclos cortos y un período anovulatorio posparto largo puede ser atribuible a un largo período de baja progesterona (es decir una falta de pre-exposición a la progesterona), en comparación con 
las vacas con ciclos de longitud normal en el que el periodo anovulatorio fue corto (no muy lejano de la caída de la progesterona placentaria). Algunos autores han sugerido que las prostaglandinas secretadas durante la involución uterina postparto pueden ser responsables de la luteólisis prematura en el período postparto (Troxel y Kesler, 1984, citado en Rajamahendran \& Taylor, 1990), pero este mecanismo no explicaría adecuadamente la asociación que existe entre la duración del intervalo posparto y la posterior función lúteal y duración del ciclo estral.

\section{La inducción de la ciclicidad posparto}

A pesar de lo mucho que se le ha dedicado en la investigación clínica, a la utilización de gonadotrofinas y esteroides para acelerar la primera ovulación posparto y ciclicidad, los resultados han sido inconsistentes (Odde, 1990). Las cuestiones importantes que han confundido los resultados de estas investigaciones incluyen el estado desconocido del desarrollo de la onda folicular en el momento del tratamiento y el uso confuso del término anestro. Como se mencionó anteriormente, el desarrollo folicular ovárico y la ovulación durante el período postparto temprano no está asociado con el estro. Además, el promedio de la eficiencia de la detección de celo es sólo del 40 al 60\% (O’Connor, 2007), por lo tanto, el anestro fisiológico no se puede separar del anestro por falta de detección. En los protocolos prácticos para el tratamiento de anestro (diseñados para ser aplicados en un día fijo después del parto), sería interesante correlacionar la respuesta ovárica a la condición del desarrollo folicular en el momento del tratamiento. Es muy probable que los efectos beneficiosos del tratamiento, reportado por algunos autores, se debe a la inducción de la ciclicidad sólo en los animales que están al borde de la ovulación espontánea, y que el tratamiento es ineficaz para inducir la ciclicidad en vacas postparto que se encuentran en un periodo anovulatorio profundo.

\section{$\backslash$ Conclusiones \}

El ciclo estral en el bovino dura normalmente entre 17 a 24 días. Durante el ciclo estral hay típicamente dos a tres ondas de crecimiento folicular que implican un periodo de emergencia, uno de selección seguido de atresia u ovulación del folículo dominante. La FSH y LH son las principales hormonas reguladoras de la foliculogénesis y la esteroidogénesis. La frecuencia de los pulsos de LH determina el destino final del folículo dominante 
(1 pulso/6-8 horas = regresión; 1 pulso/hora = ovulación). La secreción de PGF por parte del útero es la principal señal hormonal que induce la regresión del CL e interrumpe la fase luteal dando lugar a la fase folicular. La maduración final del folículo ovulatorio resulta en un incremento de la concentración plasmática de E2 que desencadena el estro y la ovulación.

\section{\Bibliografía \}

Abel, M.H; Wootton, A.N; Wilkins, V; Huhtaniemi, I; Knight, P.G; Charlton, H M. 2000. The effect of a nullmutation in the follicle-stimulating hormone receptor gene on mouse reproduction. Endocrinology, 141:1795-1803.

Adams, G.P; Sumar, J; Ginther, O.J. 1990. Effects of lactational status and reproductive status on ovarian follicular waves in llamas (Lama glama). J Reprod Fert., 90:535-545.

Adams, G.P; Kot, K; Smith, C.A; Ginther, O.J. 1993a. Selection of a dominant follicle and suppression of follicular growth in heifers. Anim Reprod Sci, 30:259-271.

Adams GP, Kot K, Smith CA, Ginther OJ. 1993b. Effect of the dominant follicle on regression of its subordinates in heifers. Can J Anim Sci, 73:267-275.

Adams, G.P; Matteri, R.L; Ginther, O.J. 1992a. The effect of progesterone on growth of ovarian follicles, emergence of follicular waves and circulating FSH in heifers. J Reprod Fert, 95:627-640.

Adams, G.P; Matteri, R.L; Kastelic, J.P; Ko, J.C.H; Ginther, O.J. 1992b. Association between surges of follicle stimulating hormone and the emergence of follicular waves in heifers. J Reprod Fert, 94:177-188.

Ahmad, N; Townsend, E.C; Dailey, R.A; Inskeep, E.K. 1997. Relationships of hormonal patterns and fertility to occurrence of two or three waves of ovarian follicles, before and after breeding, in beef cows and heifers. Anim Reprod Sci, 49:13-28.

Armstrong, D.G; Baxter, G; Gutierrez, C.G; Hogg, C.O; Glazyrin, A.L; Campbell, B.K; Bramley, T.A; Webb, R. 1998. Insulin-like growth factor binding protein -2 and -4 messenger ribonucleic acid expression in bovine ovarian follicles: effect of gonadotropins and developmental status. Endocrinology, 139:2146-2154.

Bao, B and Garverick, H.A. 1998. Expression of steroidogenic enzyme and gonadotropin receptor genes in bovine follicles during ovarian follicular waves: a review. J Anim Sci, 76:1903-1921.

Bergfelt, D.R; Kastelic, J.P; Ginther, O.J. 1991. Continued periodic emergence of follicular waves in nonbred progesterone-treated heifers. Anim Reprod Sci, 24:193-204.

Bleach, E.C.L; Glencross, R.G; Knight, P.G. 2004. Association between ovarian follicle development and pregnancy rate in dairy cows undergoing spontaneous oestrous cycles. Reproduction, 127:621-629.

Colazo, M.G and Mapletoft, R.J. 2014. A Review of Current Timed-AI (TAI) Programs for Beef and Dairy Cattle. Canadian Veterinary Journal (In press).

Evans, A.C.O; Adams, G.P; Rawlings, N.C. 1994. Endocrine and ovarian follicular changes leading up to the first ovulation in prepubertal heifers. J Reprod Fert, 100:187-194.

Farnworth, P.G. 1995. Gonadotrophin secretion revisited. How many ways can FSH leave a gonadotroph? J. Endocrinol, 145, 387-395.

Fortune, J.E; Rivera, G.M; Evans, A.C.O, Turzillo, A.M. 2001. Differentiation of dominant versus subordinate follicles in cattle. Biol Reprod, 65:648-654.

Ginther, O.J. 1974. Internal regulation of physiological processes through local ve- 
noarterial pathways: a review. J Anim Sci, 39:550-564.

Ginther, O.J; Kot, K; Kulick, L.J; Martin, S; Wiltbank, M.C. 1996a. Relationships between FSH and ovarian follicular waves during the last six months of pregnancy in cattle. J Rerpod Fert, 108:271.

Ginther, O.J; Wiltbank, M.C; Fricke, P.M; Gibbons, J.R; Kot, K. 1996b. Selection of the dominant follicle in cattle. Biol Reprod, 55:1187-1194.

Ginther, O.J; Kot, K. 1994. Follicular dynamics during the ovulatory season in goats. Theriogenology, 42: 987-1001.

Ginther, O.J. 1993. Major and minor follicular waves during the equine estrous cycle. J Equine Vet Sci., 13:18-25.

Ginther, O.J; Kastelic, J.P; Knopf, L. 1989a. Composition and characteristics of follicular waves during the bovine estrous cycle. Anim Reprod Sci., 20:187-200.

Ginther, O.J; Knopf, L; Kastelic, J.P. 1989b. Temporal associations among ovarian events in cattle during oestrous cycles with two and three follicular waves. J Reprod Fertil., 87:223-230.

Ginther, O.J; Knopf, L; Kastelic, J.P. 1989c. Ovarian follicular dynamics in heifers during early pregnancy. Biol Reprod., 41:247-254.

Gottsch, M.L; Cunningham, M.J; Smith, J.T; Popa, S.M; Acohido, B.V; Crowley, W.F; Seminara, S; Clifton; D.K; Steiner, R.A. 2004. A role for kisspeptins in the regulation of gonadotropin secretion in the mouse. Endocrinology, 145:4073-4077.

Hansel, W; Malven, P.V; Black, D.L. 1961. Estrous cycle regulation in the bovine. J Anim Sci., 20:621.

Hoare, E.K; S.E. Parker, P.F; Flood and G.P. Adams. 1997. Ultrasonic imaging of reproductive events in muskoxen. Rangifer, 17: $119-123$.

Inskeep EK, Braden TD, Lewis PE, Garcia-Winder M, Niswender GD. 1988. Receptors for luteinizing hormone and follicle stimulating hormone in largest follicles of postpartum beef cows. Biol Reprod., 38:587-591.

Kakar, S.S; Rahe, C.H; Neill, J.D. 1993. Molecular cloning, sequencing, and characterizing the bovine receptor for gonadotropin releasing hormone $(\mathrm{GnRH})$. Domest. Anim. Endocrinol., 10, 335-342.

Kastelic, J.P; Ginther, O.J. 1991. Factors affecting the origin of the ovulatory follicle in heifers with induced luteolysis. Anim Reprod Sci., 26:13-24.

Kastelic, J.P; Knopf, L; Ginther, O.J. 1990. Effect of day of prostaglandin $\mathrm{F}_{2 \mathrm{a}}$ treatment on selection and development of the ovulatory follicle in heifers. Anim Reprod Sci., 23:169-180.

Kastelic, J.P; Mapletoft, R.J; Colazo, M.G; Martinez, M.F. 2008. Ovarian follicular development and synchronization of estrus and ovulation in cattle. World Buiatrics Congress in Budapest, Hungary, July 2008.

Kinder, J.E; Kojima, F.N; Bergfeld, E.G.M; Wehrman, M.E; Fike, K.E. 1996. Progestin and estrogen regulation of pulsatile LH release and development of persistent ovarian follicles in cattle. J Anim Sci., 74:1424-1440.

Knopf, L; Kastelic, J.P; Schallenberger, E; Ginther, O.J. 1989. Ovarian follicular dynamics in heifers: test of two-wave hypothesis by ultrasonically monitoring individual follicles. Dom Anim Endocr., 6:111-119.

Ko, J.C.H; Kastelic, J.P; Del Campo, M.R; Ginther, O.J. 1991. Effects of a dominant follicle on ovarian follicular dynamics during the oestrous cycle in heifers. J Reprod Fert., 91:511-519.

Larson L.L, Ball, P.J.H. 1992. Regulation of estrous cycles in dairy cattle: a review. Theriogenology, 38: 255-267.

Lussier, J.G; Matton, P; Dufour, J.J. 1987. Growth rates of follicles in the ovary of the cow. J Reprod Fertil., 81:301-307.

Macmillan, K.L. 2010. Recent advances in the synchronization of estrus and ovulation in dairy cows. J Reprod Dev., 56:42-47. 
Macmillan, K.L; Segwagwe, B.V.E; Pino, C.S. 2003. Associations between the manipulation of patterns of follicular development and fertility in cattle. Anim Reprod Sci., 78: 327-344.

Mapletoft, R.J; Colazo, M.G; Martínez, M.F; Kastelic, J.P. 2002. Estrogen esters to synchronize follicular wave emergence and ovulation in CIDR-treated cattle. Proceedings of the Annual Convention of the American Embryo Transfer Association, Pag. 27-38.

McCracken, J A. 1972. Prostaglandins and luteal regression - A review. Prostaglandins, 1:1-4.

McNatty, K.P; Heath, D.A; Lundy, T; Fidler, A.E; Quirke, L; O’Connell, A; Smith, P; Groome, N; Tisdall, D.J. 1999. Control of early ovarian follicular development. J Reprod Fertil., Suppl 54:3-16.

Mihm, M and Bleach, E.C. 2003. Endocrine regulation of ovarian antral follicle development in cattle. Anim Reprod Sci., 78:217-237.

Mihm, M; Austin, E.J; Good, T.E.M; Ireland, J.L.H; Knight, P.G; Roche, J.F; Ireland, J.J. 2000. Identification of potential intrafollicular factors involved in selection of dominant follicles in heifers. Biol Reprod., 63:811-819.

Moenter, S.M; Brand, R.C; Karsch, F.J. 1992. Dynamics of gonadotropin releasing hormone $(\mathrm{GnRH})$ secretion during the GnRH surge: insights into the mechanism of GnRH surge induction. Endocrinology 130, 2978-2984.

Murphy, M.G; Boland, M.P; Roche, J.F. 1990. Pattern of follicular growth and resumption of ovarian activity in post-partum beef suckle cows. J Reprod Fert., 90:523-533.

Murphy, M.G; Enright, W.J; Crowe, M.A; McConnell, K; Spicer, L.J; Boland, M.P; Roche, J.F. 1991. Effect of dietary intake on pattern of growth of dominant follices during the estrous cycle in beef heifers. J Reprod Fert., 92:333-338.

Nett, T.M; Crowder, M.E; Wise, M.E. 1984. The role of estradiol in inducing an ovulatory- like surge of LH in sheep. Biol Reprod., 30:1208-1215.

Niswender, G.D; Juengel, J.L; Silva, P.J; Rollyson, M.K; McIntush, E.W. 2000. Mechanisms controlling the function and life span of the corpus luteum. Physiol Rev., 80:1-29.

O’Connor, M.L. 2007. Estrus detection. In: Youngquist RS, Threlfall WR editors. Large Animal Theriogenology 2. St. Louis, Missouri. Saunders:270-278.

Odde, K.G. 1990. A review of synchronization of estrus in postpartum cattle. J Anim Sci., 68:817-830.

Pierson, R.A and Ginther, O.J. 1984. Ultrasonography of the bovine ovary. Theriogenology, 21:495-504.

Rahe, C.H; Owens, R.E; Fleeger, J.L; Newton, H.J; Harms, P.G. 1980. Pattern of plasma luteinizing hormone in the cyclic cow: dependence upon the period of the cycle. Endocrinology, 107:498-503.

Rajakoski, E. 1960. The ovarian follicular system in sexually mature heifers with special reference to seasonal, cyclical, and left-right variations. Acta Endocrinol., 34:7-68.

Rajamahendran, R; Taylor, C. 1990. Characterization of ovarian activity in postpartum dairy cows using ultrasound imaging and progesterone profiles. Anim Reprod Sci., 22: 171-180.

Ravindra, J.P; Rawlings, N.C; Evans, A.C.O; Adams, G.P. 1994. Ultrasonographic study of ovarian follicular dynamics in ewes during the oestrous cycle. J Reprod Fert., 101:501509 .

Rhodes, J.M; De'ath, G; Entwistle, K.W. 1995. Animal and temporal effects on ovarian follicular dynamics in Brahman heifers. Anim Reprod Sci., 38:265-277.

Roche, J.F; Boland, M.P. 1991. Turnover of dominant follicles in cattle of different reproductive states. Theriogenology, 35:81-90.

Roche, J.F; Mihm, M; Diskin, M.G. 1997. Physiology and practice of induction and 
control of oestrus in cattle. The Bovine Practitioner, 31.2:4-10.

Sartori R, Barros C.M. 2011. Reproductive cycles in Bos indicus cattle. Anim Reprod Sci., 124:244-250.

Savio, J.D; Keenan, L; Boland, M.P; Roche, J.F. 1988. Pattern of growth of dominant follicles during the oestrous cycle of heifers. J Reprod. Fert., 83:663-671.

Savio, J.D; Boland, M.P; Roche, J.F. 1990a. Development of dominant follicles and length of ovarian cycles in postpartum dairy cows. J Reprod Fert., 88:581-591.

Savio, J.D, Boland, M.P; Hynes, N; Roche, J.F. 1990b. Resumption of follicular activity in the early postpartum period of dairy cows. J Reprod Fert., 88:569-579.

Savio, J.D; Thatcher, W.W; Badinga, L; de la Sota, R.L; Wolfenson, D. 1993. Regulation of dominant follicle turnover during the oestrous cycle in cows. J Reprod. Fert., 97:197-203.

Sirois, J; Fortune, J.E. 1988. Ovarian follicular dynamics during the estrous cycle in heifers monitored by real-time ultrasonography. Biol. Reprod., 39:308-317.

Sirois, J; Fortune, J.E. 1990. Lengthening the bovine estrous cycle with low levels of exogenous progesterone: a model for studying ovarian follicular dominance. Endocrinology, 127:916-925.

Smith, V.G; Chenault, J.R; McAllister, J.F; Lauderdale, J.W. 1987. Response of postpartum beef cows to exogenous progestogens and gonadotropin releasing hormone. J Anim Sci., 64:540-551.

Spicer, L.J and Echternkamp, S.E. 1995. The ovarian insulin and insulin-like growth factor system with emphasis on domestic animals. Domest Anim Endocrinol., 12: 223-245.
Stevenson JS. 2007. Clinical reproductive physiology. In: Youngquist RS, Threlfall WR editors. Large Animal Theriogenology 2. St. Louis, Missouri: Saunders, 258-270.

Stock, A.E; Fortune, J.E. 1993. Ovarian follicular dominance in cattle: Relationship between prolonged growth of the ovulatory follice and endocrine parameters. Endocrinology, 132:1108-1114.

Townson, D.H; Tsang, P.C; Butler, W.R; Frajblat, M; Griel, L.C Jr, Johnson, C.J; Milvae, R.A; Niksic, G.M; Pate, J.L. 2002. Relationship of fertility to ovarian follicular waves before breeding in dairy cows. J Anim Sci., 80:1053-1058.

Troxel, T.R; Kesler, D.J. 1984. The effect of progestin and $\mathrm{GnRH}$ treatments on ovarian function and reproductive hormone secretions of anestrous post-partum sucked beef cows. Theriogenology, 21:699-711.

Wiltbank, M.C. 1997. How information of hormonal regulation of the ovary has improved understanding of timed breeding programs. Proceedings of the Annual Meeting of the Society for Theriogenology. Pp. 83-97.

Xu, Z.Z; Garverick, H.A; Smith, G.W; Smith, M.F; Hamilton, S.A; Youngquist, R.S. 1995. Expression of follicle-stimulating hormone and luteinizing hormone receptor messenger ribonucleic acids in bovine follicles during the first follicular wave. Biol Reprod, 53:951-957.

Zeitoun, M.M; Rodriguez, H.F; Randel, R.D. 1996. Effect of season on ovarian follicular dynamics in Brahman cows. Theriogenology, 45:1577-1581. 